\title{
THE ESTIMATION OF MATERIAL AND PATCH PARAMETERS IN A PDE-BASED CIRCULAR PLATE MODEL ${ }^{1}$
}

\author{
H.T. Banks \\ Center for Research in Scientific Computation \\ North Carolina State University \\ Raleigh, NC 27695 \\ D.E. Brown \\ Lockheed Eng. and Sciences Company \\ NASA Langley Research Center \\ Hampton, VA 23681
}

\author{
Ralph C. Smith \\ Department of Mathematics \\ Iowa State University \\ Ames, IA 50011 \\ Vern L. Metcalf \\ U.S. Army Research Laboratory \\ NASA Langley Research Center \\ Hampton, VA 23681
}

\author{
R.J. Silcox \\ Acoustics Division \\ NASA Langley Research Center \\ Hampton, VA 23681
}

\begin{abstract}
The estimation of material and patch parameters for a system involving a circular plate, to which piezoceramic patches are bonded, is considered. A partial differential equation (PDE) model for the thin circular plate is used with the passive and active contributions from the patches included in the internal and external bending moments. This model contains piecewise constant parameters describing the density, flexural rigidity, Poisson ratio and Kelvin-Voigt damping for the system as well as patch constants and a coefficient for viscous air damping. Examples demonstrating the estimation of these parameters with experimental acceleration data and a variety of inputs to the experimental plate are presented. By using a physicallyderived PDE model to describe the system, parameter sets consistent across experiments are obtained, even when phenomena such as damping due to electric circuits affect the system dynamics.
\end{abstract}

\footnotetext{
${ }^{1}$ The research of H.T.B. was supported in part by the Air Force Office of Scientific Research under grant AFOSR-F49620-93-1-0198. This research was also supported by the National Aeronautics and Space Administration under NASA Contract Number NAS1-19480 while H.T.B. and R.C.S. were visiting scientists at the Institute for Computer Applications in Science and Engineering (ICASE), NASA Langley Research Center, Hampton, VA 23681. Additional support for H.T.B. and R.C.S. was also provided in part under NASA grant NAG-1-1600. Finally, the research of D.E.B. was supported under NASA Contract Number NAS1-19000.
} 


\section{Introduction}

In many applications involving vibrating structures, it is both feasible and advantageous to start with basic physical principles and from them, derive a PDE model describing the system dynamics. Such models can directly incorporate the effects of nonhomogeneities, actuators or sensors bonded to or embedded in the structure (e.g., piezoceramic patches), coupling with adjacent acoustic or fluid fields if they exist, and contributions due to multiple components, inexact boundary conditions, and any other influences which may affect the system dynamics. General constitutive laws, moment and force relations, and electromechanical laws are used when deriving these models. This then leads to PDE models having physical parameters which must be estimated through fit-to-data techniques.

Consider, for example, a structural system with surface-mounted piezoelectric actuators and sensors. Physical parameters include density, stiffness, damping and Poisson ratios for the structure and electromechanical coefficients describing the strain generated and sensed by the piezoelectric elements. These parameters must be determined for the experimental system under consideration before the PDE models can be used with any accuracy in model-based applications such as simulation or control. In simulations, the use of inexact parameters can lead to spurious results, whereas controllers will be degraded or even potentially destabilized by the use of inexact parameter values.

While "handbook" values often exist for the density, stiffness and Poisson coefficients for the material in a uniform and homogeneous structure, they usually cannot be used with certainty or reliability in the models describing actual experimental structures due to nonhomogeneities in materials, and differing geometries and material properties in the regions of actuators and sensors. Similarly, while electromechanical constants for actuators and sensors can often be found in manufacturer specifications, variability in actual experimental conditions necessitates the estimation of these parameters before PDE models can be employed in simulation and control applications. Finally, the estimation of damping coefficients using experimental data is crucial since accurate compilations of damping coefficients for various materials do not exist.

Several studies regarding the estimation of parameters in PDE models for homogeneous beams [11, 12], plates [5] and grid structures [3, 4] have been reported. Furthermore, results pertaining to parameter estimation issues which arise when piezoceramic patches are used as sensors and actuators on a beam can be found in $[13,14]$. There it was demonstrated that the stiffness, density and damping parameters for a beam with surface-mounted piezoceramic actuators and sensors must be taken to be piecewise-constant to account for the differing geometry and material properties of the patches. When this was done, consistency across experiments with a variety of inputs and outputs was obtained, thus validating the applicability of the PDE model for the system.

In this work, we extend the PDE-based parameter estimation methods of [14] to a clamped circular plate with surface-mounted piezoceramic patches. The dynamics of the plate differ from those of a beam in that Poisson effects provide a coupling between the radial and tangential vibrations. Hence, when estimating parameters for the plate, one must work with data containing significantly more frequencies than is typically the case with the beams. The results reported here differ from those in $[3,4,5]$ in that the emphasis here is on the consistent estimation of physical parameters for a plate whose dynamics are influenced by the presence and excitation of piezoceramic patches. 
In addition to issues regarding the estimation of the density, stiffness, Poisson and material and air damping parameters, questions concerning the passive damping due to the patches are addressed. It is well-known among experimentalists that significant passive damping is provided when the circuit for the piezoceramic patch is closed or shunted [16]. This general phenomenon for structures is investigated in the context of the circular plate with its corresponding PDEbased model.

By using PDE models and estimating parameters through fit-to-data techniques, model fits to data that are consistent across experiments are obtained, even in the presence of passive patch damping. Up to six axisymmetric and eight nonaxisymmetric frequencies are matched through time-domain optimization, thus demonstrating that the effectiveness of the the model is not dependent upon the number of excited frequencies. The distributed nature of the model is further demonstrated by examples illustrating the match of the model response with data measured at plate points not used in the optimization process. As discussed in [1], the model, with parameters estimated from experimental data, is sufficiently accurate so as to be very effective when incorporated in PDE-based control methods for reducing plate vibrations.

We note that throughout this work, fixed-edge (zero displacement and slope) boundary conditions are assumed. This assumption was made after tests indicated minimal energy loss through the boundary clamps. In many structures, however, boundary movement makes this assumption inappropriate. In such cases, a boundary moment model of the type discussed in [9] and experimentally investigated in [2] may provide a more accurate description of the boundary physics.

In Section 2, the strong and weak forms of the PDE model for a thin circular plate with fixededge boundary conditions are discussed. Care is taken to include both the passive and active contributions due to the piezoceramic patches when developing this model. A Fourier-Galerkin approximation method and the parameter estimation problem are outlined in Section 3 . A modified cubic spline basis in the radial direction provides accurate approximates and facilitates the incorporation of patch effects. The final section of the paper contains a repertoire of examples demonstrating the model fits when parameters are estimated in a variety of experiments. These examples demonstrate the accuracy of the PDE model for describing the plate dynamics and the effects of passive damping due to the shunted patches.

\section{$2 \quad$ Plate Model}

The structure under consideration consists of a thin circular plate mounted to a frame with a heavy metal collar. Bonded to the plate are piezoceramic patches which are mounted either individually or in pairs as illustrated in Figure 1. As discussed in [8], the free patches generate strains in response to an applied voltage. When bonded to an underlying structure, these strains lead to the generation of in-plane forces and/or bending moments as depicted in Figure 2. In this paper, we will consider only the bending moments which are generated by the patches and will consider them as an input to a model describing the transverse vibrations of a plate. 

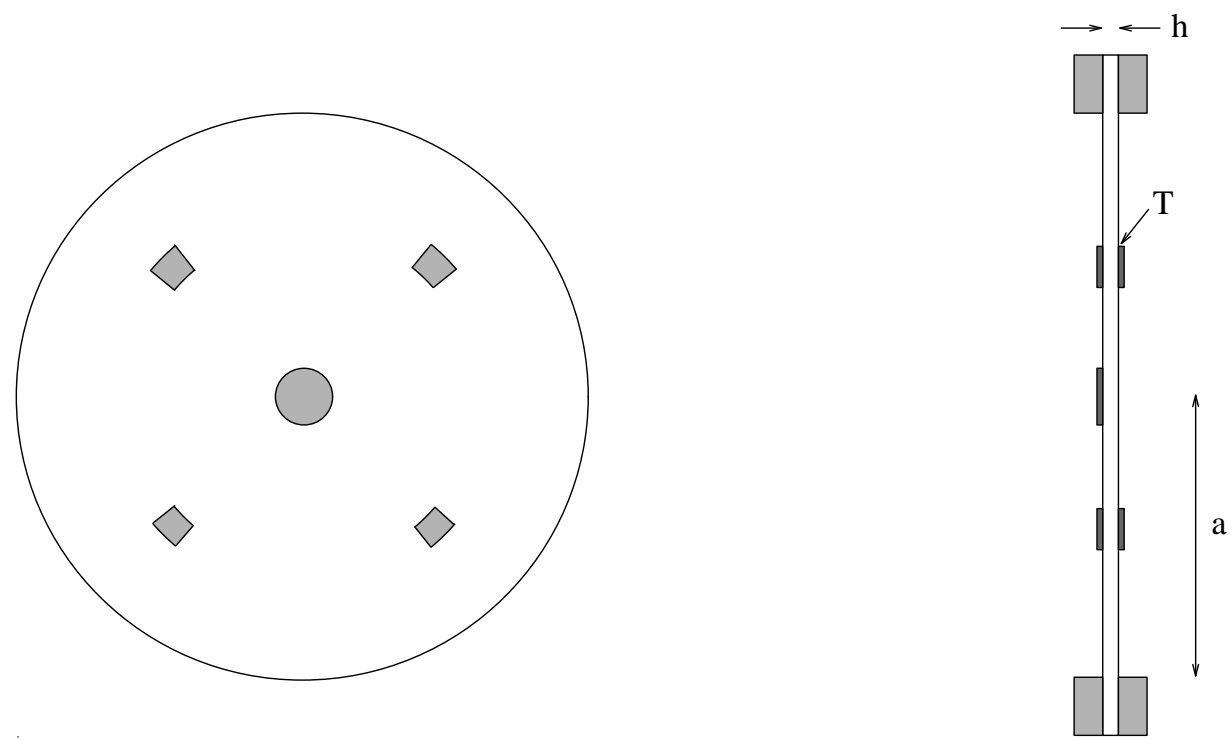

Figure 1. A thin circular plate with piezoeceramic patches bonded individually or in pairs to its surface.

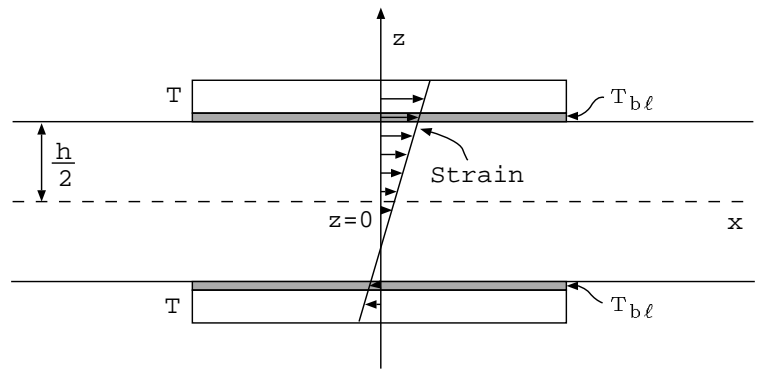

(a)

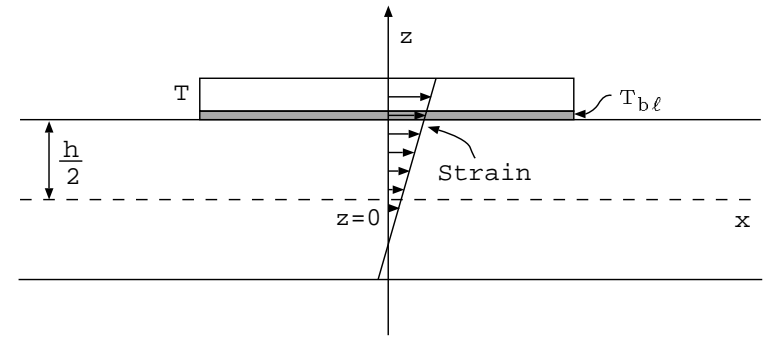

(b)

Figure 2. Strain distribution resulting from applied voltages to (a) a patch pair, and (b) a single piezoceramic patch. In both cases, in-plane forces and/or bending moments can be generated.

\subsection{Strong Form of the Plate Equation}

For this discussion, we will consider a plate of radius $a$ and thickness $h$ as shown in Figure 1. The radial and circumferential coordinates are denoted by $r$ and $\theta$, respectively. Bonded to the plate are patches of thickness $T$ with a bonding layer that is assumed to have uniform thickness $T_{b \ell}$ (see Figure 2). The Young's modulus, density coefficient, Poisson ratio and KelvinVoigt damping coefficient for the plate are denoted by $E_{p}, \rho_{p}, \nu_{p}$ and $\hat{c}_{D_{p}}$, respectively, while similar parameters for the patches and bonding layer are denoted by $E_{p e}, \nu_{p e}, \nu_{p e}, \hat{c}_{D_{p e}}$ and $E_{b \ell}, \nu_{b \ell}, \nu_{b \ell}, \hat{c}_{D_{b \ell}}$, respectively. We point out that the assumption that all the patches (and respectively, bonding layers) have the same Young's modulus and Poisson ratio is made only 
for ease of exposition and analogous formulations result when differing values are assumed for the individual patches and bonding layers (see, for example, [8]).

Letting $w, \gamma, \rho$ and $f$ denote, respectively, the transverse plate displacement, viscous air damping coefficient, density for the combined structure, and external surface force, the strong form of the equations modeling the transverse motion is

$$
\rho h \frac{\partial^{2} w}{\partial t^{2}}+\gamma \frac{\partial w}{\partial t}-\frac{\partial^{2} \mathcal{M}_{r}}{\partial r^{2}}-\frac{2}{r} \frac{\partial \mathcal{M}_{r}}{\partial r}+\frac{1}{r} \frac{\partial \mathcal{M}_{\theta}}{\partial r}-\frac{2}{r} \frac{\partial^{2} \mathcal{M}_{r \theta}}{\partial r \partial \theta}-\frac{2}{r^{2}} \frac{\partial \mathcal{M}_{r \theta}}{\partial \theta}-\frac{1}{r^{2}} \frac{\partial^{2} \mathcal{M}_{\theta}}{\partial \theta^{2}}=f(t, r, \theta)
$$

with initial conditions

$$
w(0, r, \theta)=w_{0}(r, \theta) \quad, \quad \frac{\partial w}{\partial t}(0, r, \theta)=w_{1}(r, \theta) .
$$

The general moments are given by

$$
\begin{aligned}
\mathcal{M}_{r} & =M_{r}-\left(M_{r}\right)_{p e} \\
\mathcal{M}_{\theta} & =M_{\theta}-\left(M_{\theta}\right)_{p e} \\
\mathcal{M}_{r \theta} & =M_{r \theta}
\end{aligned}
$$

where $M_{r}, M_{\theta}$ and $M_{r \theta}$ are internal plate moments, and $\left(M_{r}\right)_{p e}$ and $\left(M_{\theta}\right)_{p e}$ are the external moments generated by the patches. The internal moments for the circular plate with $s$ pairs of surface-mounted piezoceramic patches are

$$
\begin{aligned}
& M_{r}=D K_{r}+\tilde{D} K_{\theta}+c_{D} \dot{K}_{r}+\tilde{c}_{D} \dot{K}_{\theta} \\
& M_{\theta}=D K_{\theta}+\tilde{D} K_{r}+c_{D} \dot{K}_{\theta}+\tilde{c}_{D} \dot{K}_{r} \\
& M_{r \theta}=M_{\theta r}=\frac{D}{2} \tau-\frac{\tilde{D}}{2} \tau+\frac{c_{D}}{2} \dot{\tau}-\frac{\tilde{c}_{D}}{2} \dot{\tau}
\end{aligned}
$$

where

$$
K_{r}=-\frac{\partial^{2} w}{\partial r^{2}} \quad, \quad K_{\theta}=-\frac{1}{r} \frac{\partial w}{\partial r}-\frac{1}{r^{2}} \frac{\partial^{2} w}{\partial \theta^{2}} \quad, \quad \tau=-\frac{2}{r} \frac{\partial^{2} w}{\partial r \partial \theta}+\frac{2}{r^{2}} \frac{\partial w}{\partial \theta} .
$$

The global flexural rigidity parameters $D, \tilde{D}$ and Kelvin-Voigt damping parameters $c_{D}$ and $\tilde{c}_{D}$ are given by

$$
\begin{aligned}
& D(r, \theta)=\frac{E_{p} h^{3}}{12\left(1-\nu_{p}^{2}\right)}+\frac{2}{3} \sum_{i=1}^{s}\left[\frac{E_{p e} a_{3 p e}}{1-\nu_{p e}^{2}}+\frac{E_{b \ell} a_{3 b \ell}}{1-\nu_{b \ell}^{2}}\right] \chi_{i}(r, \theta) \\
& \tilde{D}(r, \theta)=\frac{E_{p} h^{3} \nu_{p}}{12\left(1-\nu_{p}^{2}\right)}+\frac{2}{3} \sum_{i=1}^{s}\left[\frac{E_{p e} a_{3 p e} \nu_{p e}}{1-\nu_{p e}^{2}}+\frac{E_{b \ell} a_{3 b \ell} \nu_{b \ell}}{1-\nu_{b \ell}^{2}}\right] \chi_{i}(r, \theta) \\
& c_{D}(r, \theta)=\frac{\hat{c}_{D_{p}} h^{3}}{12\left(1-\nu_{p}^{2}\right)}+\frac{2}{3} \sum_{i=1}^{s}\left[\frac{\hat{c}_{D_{p e}} a_{3 p e}}{1-\nu_{p e}^{2}}+\frac{\hat{c}_{D_{b \ell}} a_{3 b \ell}}{1-\nu_{b \ell}^{2}}\right] \chi_{i}(r, \theta) \\
& \tilde{c}_{D}(r, \theta)=\frac{\hat{c}_{D_{p}} h^{3} \nu_{p}}{12\left(1-\nu_{p}^{2}\right)}+\frac{2}{3} \sum_{i=1}^{s}\left[\frac{\hat{c}_{D_{p e}} a_{3 p e} \nu_{p e}}{1-\nu_{p e}^{2}}+\frac{\hat{c}_{D_{b \ell}} a_{3 b \ell} \nu_{b \ell}}{1-\nu_{b \ell}^{2}}\right] \chi_{i}(r, \theta) .
\end{aligned}
$$


Here $a_{3 b \ell}=\left(h / 2+T_{b \ell}\right)^{3}-(h / 2)^{3}, a_{3 p e}=\left(h / 2+T_{b \ell}+T\right)^{3}-\left(h / 2+T_{b \ell}\right)^{3}$ and $\chi_{i}(r, \theta)$ denotes the characteristic function which has a value of 1 in the region covered by the $i^{\text {th }}$ patch and is 0 elsewhere. A similar definition is used for the density which also exhibits a piecewise constant nature due to the presence of the patches. These definitions can be adapted to the case of a single patch that is bonded to the plate by replacing the $2 / 3$ by $1 / 3$. We point out that if the plate, patches and bonding layers have the same Poisson ratios $\left(\nu_{p}=\nu_{p e}=\nu_{b l}=\nu\right)$, then the internal moment expressions reduce to the familiar relations for a thin plate. For example, $M_{r}$ in this case is given by

$$
M_{r}=-D\left(\frac{\partial^{2} w}{\partial r^{2}}+\frac{\nu}{r} \frac{\partial w}{\partial r}+\frac{\nu}{r^{2}} \frac{\partial^{2} w}{\partial \theta^{2}}\right)-c_{D}\left(\frac{\partial^{3} w}{\partial r^{2} \partial t}+\frac{\nu}{r} \frac{\partial^{2} w}{\partial r \partial t}+\frac{\nu}{r^{2}} \frac{\partial^{3} w}{\partial \theta^{2} \partial t}\right)
$$

with $D$ and $c_{D}$ defined in (2.3).

The external moments generated by the patches in response to an applied voltage (out-ofphase for the patch pair) are given by

$$
\left(M_{r}\right)_{p e}=\left(M_{\theta}\right)_{p e}=-\sum_{i=1}^{s} \mathcal{K}_{i}^{B} u_{i}(t) \chi_{i}(r, \theta)
$$

where $u_{i}(t)$ is the voltage into the $i^{t h}$ patch (or patch pair) and $\mathcal{K}_{i}^{B}$ is a parameter given by

$$
\mathcal{K}_{i}^{B}= \begin{cases}\frac{1}{2} \cdot \frac{E_{p e}}{1-\nu_{p e}} d_{31}\left(h+2 T_{b \ell}+T\right) & , \text { active single patch } \\ \frac{E_{p e}}{1-\nu_{p e}} d_{31}\left(h+2 T_{b \ell}+T\right) & , \text { active patch pair }\end{cases}
$$

(see [8] for details). In these expressions, the piezoelectric strain constant $d_{31}$ relates the input voltage to the free strain generated in the patch.

In the case of perfectly clamped edges, zero displacement and slope are maintained at the plate perimeter and the boundary conditions are taken to be

$$
w(t, a, \theta)=\frac{\partial w}{\partial r}(t, a, \theta)=0
$$

For the experiments discussed here, this fixed-edge boundary condition adequately modeled the edge dynamics and hence it is used throughout this work. In many applications, however, perfect clamps modeled by fixed-edge boundary conditions are difficult to attain, thus resulting in frequencies that are lower than expected $[17,18,21,22]$. In such cases, boundary moment models of the type discussed in $[9,10]$ can be employed.

We point out that the piezoceramic material parameters $\mathcal{K}_{i}^{B}, i=1, \cdots, s$ and the plate parameters $\rho, D, \nu, c_{D}$ and $\gamma$ should be considered as unknown and in applications must be estimated using the fit-to-data techniques to be discussed in the next section $\left(\tilde{D}\right.$ and $\tilde{c}_{D}$ can be constructed using components of $D, c_{D}$ and $\nu$ ). One might argue that in regions of the plate not covered by patches, "handbook" values of $\rho, D$ and $\nu$ for the aluminum plate material be used and parameter estimation avoided. As demonstrated by the examples, however, there exists sufficient variation in material properties (and boundary conditions) so as to yield plate parameters which vary significantly from the "handbook" values. Similarly, manufacturer specified 
values for $d_{31}$ can be found for various piezoceramic materials and hence the analytic moments generated by the patches can be obtained from (2.4). The specified values for $d_{31}$ can vary by batch, however, and the static values listed by manufacturers are often significantly larger than the actual values obtained in dynamic experiments [15, 19, 20]. Finally, the strain output for the patches often decays over time which is manifested in the moment expression (2.4) by a decrease in $d_{31}$. Hence, all of the parameters listed above must be estimated before an accurate fit of the model to the experimental system can be expected.

\subsection{Weak Form of the Plate Equations}

Due to the piecewise constant nature of the physical parameters $D, \nu$ and $c_{D}$, one is forced to differentiate discontinuous functions when considering the strong form of the plate equations (2.1). Moreover, the input due to the excitation of the patches is spatially discontinuous since it is defined only in the regions of the active patches. Since this input acts as a bending moment on the plate, it too is twice differentiated when considering the strong form of the plate equations, thus yielding a distribution having the regularity of a differentiated Dirac delta "function." To avoid these difficulties as well as lower smoothness requirements for approximating elements, we will consider a weak form of the modeling plate equations.

We begin by defining appropriate spaces in which to consider the evolution and approximation of the plate dynamics. For a plate having perfectly clamped edges and hence boundary conditions (2.6), the state for the problem is taken to be the transverse displacement $w$ in the state space $H=L^{2}\left(\Gamma_{0}\right)$ where $\Gamma_{0}$ denotes the region occupied by the unstrained neutral surface of the plate. Motivated by the energy considerations discussed in [7], we also define the space of test functions $V=H_{0}^{2}\left(\Gamma_{0}\right) \equiv\left\{\eta \in H^{2}\left(\Gamma_{0}\right) \mid \eta(a, \theta)=\frac{\partial \eta}{\partial r}(a, \theta)=0\right\}$.

A weak or variational form of the equation describing the transverse motion of a damped thin circular plate having perfectly clamped edges and $s$ surface-mounted piezoceramic patches or patch pairs is then

$$
\begin{aligned}
\int_{\Gamma_{0}} \rho h \frac{\partial^{2} w}{\partial t^{2}} \bar{\eta} d \omega & +\int_{\Gamma_{0}} \gamma \frac{\partial w}{\partial t} \bar{\eta} d \omega-\int_{\Gamma_{0}} M_{r} \frac{\overline{\partial^{2} \eta}}{\partial r^{2}} d \omega-\int_{\Gamma_{0}} \frac{1}{r^{2}} M_{\theta}\left[r \overline{\frac{\partial \eta}{\partial r}}+\overline{\frac{\partial^{2} \eta}{\partial \theta^{2}}}\right] d \omega \\
& -2 \int_{\Gamma_{0}} \frac{1}{r^{2}} M_{r \theta}\left[r \frac{\partial^{2} \eta}{\partial r \partial \theta}-\overline{\frac{\partial \eta}{\partial \theta}}\right] d \omega \\
& =-\int_{\Gamma_{0}} \sum_{i=1}^{s} \mathcal{K}_{i}^{B} u_{i}(t) \chi_{i}(r, \theta) \overline{\nabla^{2} \eta} d \omega+\int_{\Gamma_{0}} f \bar{\eta} d \omega
\end{aligned}
$$

for all test functions $\eta \in V$. The overbar here denotes complex conjugation and the differential is $d \omega=r d \theta d r$.

We point out that in the weak form, the derivatives are transferred from the moments onto the test functions, thus eliminating the difficulties associated with the differentiation of discontinuous physical parameters and patch input terms. This is then an appropriate form in which to approximate the plate dynamics and consider parameter identification techniques to estimate the unknown physical parameters. For the interested reader, further details concerning the development and well-posedness of this model can be found in $[6,7]$. 


\section{System Approximation and Parameter Estimation}

In order to develop techniques for numerically simulating plate dynamics, estimating parameters and implementing control schemes, one must approximate the infinite dimensional states and test functions in (2.7). In doing so, care must be taken at the origin to avoid numerical instabilities and decreased convergence rates due to the coordinate singularity (as manifested by the $\frac{1}{r}$ and $\frac{1}{r^{2}}$ terms in the moment expressions). The approach used here follows that described in $[7,23]$.

\subsection{Approximate Plate Solution and Resulting Matrix System}

As discussed in [7, 23], an appropriate choice for the basis and Fourier-Galerkin expansion of the plate displacement is $B_{k}^{\mathcal{N}}(r, \theta)=r^{|\hat{m}|} B_{n}^{m}(r) e^{i m \theta}$ and

$$
w^{\mathcal{N}}(t, r, \theta)=\sum_{m=-M}^{M} \sum_{n=1}^{N^{m}} w_{m n}^{\mathcal{N}}(t) r^{|\hat{m}|} B_{n}^{m}(r) e^{i m \theta}=\sum_{k=1}^{\mathcal{N}} w_{k}^{\mathcal{N}}(t) B_{k}^{\mathcal{N}}(r, \theta) .
$$

Here $B_{n}^{m}(r)$ is the $n^{t h}$ modified cubic spline satisfying $B_{n}^{m}(a)=\frac{d B_{n}^{m}(a)}{d r}=0$ with the condition $\frac{d B_{n}^{m}(0)}{d r}=0$ being enforced when $m=0$ (this latter condition guarantees differentiability at the origin and implies that

$$
N^{m}= \begin{cases}N & , m=0 \\ N+1 & , m \neq 0\end{cases}
$$

where $N$ denotes the number of modified cubic splines). The total number of plate basis functions is $\mathcal{N}=(2 M+1)(N+1)-1$. As discussed in the $[7,23]$, the inclusion of the weighting term $r^{|\hat{m}|}$ with

$$
\hat{m}= \begin{cases}0 & , m=0 \\ 1 & , m \neq 0\end{cases}
$$

is motivated by the asymptotic behavior of the Bessel functions (which make up the analytic plate solution) as $r \rightarrow 0$. It also serves to ensure the uniqueness of the solution at the origin. The Fourier coefficient in the weight is truncated to control the conditioning of the mass and stiffness matrices (see the examples in [7]).

To obtain a matrix system, the $\mathcal{N}$ dimensional approximating subspace is taken to be $H^{\mathcal{N}}=\operatorname{span}\left\{B_{k}^{\mathcal{N}}\right\}$ and the product space for the first-order system is $H^{\mathcal{N}} \times H^{\mathcal{N}}$. The restriction of the infinite-dimensional system $(2.7)$ to the space $H^{\mathcal{N}} \times H^{\mathcal{N}}$ then yields a matrix system of the form

$$
\begin{aligned}
& \dot{y}^{\mathcal{N}}(t)=A^{\mathcal{N}} y^{\mathcal{N}}(t)+B^{\mathcal{N}} u(t)+F^{\mathcal{N}}(t) \\
& y^{\mathcal{N}}(0)=y_{0}^{\mathcal{N}}
\end{aligned}
$$

where $y^{\mathcal{N}}(t)=\left[w_{1}(t), \cdots, w_{\mathcal{N}}(t), \dot{w}_{1}(t), \cdots, \dot{w}_{\mathcal{N}}(t)\right]$ denotes the $2 \mathcal{N}$ column vector containing the generalized Fourier coefficients for the approximate displacement and velocity. Details concerning the construction of the component vectors and matrices in (3.2) can be found in $[7$, 23]. In this form, the finite-dimensional parameter estimation problem can be readily discussed. 


\subsection{Parameter Estimation}

The parameter estimation problem is posed as the problem of determining estimates of the "true" physical parameters $\rho, D, \nu, c_{D}, \gamma, \mathcal{K}_{1}^{B}, \cdots, \mathcal{K}_{s}^{B}$ given data measurements $z$. In the experimental results reported here, this data consisted of time histories of the transverse plate acceleration which were obtained from accelerometers located at various coordinates on the plate.

As discussed previously, the parameters $\rho, D, \nu$ and $c_{D}$ are assumed to be piecewise constants in order to account for the presence and differing material properties of the piezoceramic patches. For the case in which $s$ patches or patch pairs are bonded to the plate, these parameters can then be expressed as

$$
\begin{aligned}
& \rho(r, \theta)=\sum_{i=1}^{s+1} c_{\rho i} \chi_{i}(r, \theta) \quad, \quad D(r, \theta)=\sum_{i=1}^{s+1} c_{D i} \chi_{i}(r, \theta) \\
& \nu(r, \theta)=\sum_{i=1}^{s+1} c_{\nu i} \chi_{i}(r, \theta) \quad, \quad c_{D}(r, \theta)=\sum_{i=1}^{s+1} c_{c_{D} i} \chi_{i}(r, \theta)
\end{aligned}
$$

where again, $\chi_{i}(r, \theta), i=1, \cdots, s$ is the characteristic functions over the $i^{\text {th }}$ patch or patch pair and $\chi_{s+1}$ is the characteristic function over the portion of the plate not covered with patches. The damping due to air is assumed to be uniform over the entire surface; hence $\gamma$ is taken to be constant. Moreover, we recall from the definition $(2.5)$ that the patch parameters $\mathcal{K}_{1}^{B}, \cdots, \mathcal{K}_{s}^{B}$ are constants which depend on piezoelectric properties, the geometry and size of the patch, and bonding layer and patch properties.

To formulate the problem in an optimization setting, we let $q=\left(\rho, D, \nu, c_{D}, \gamma, \mathcal{K}_{1}^{B}, \cdots, \mathcal{K}_{s}^{B}\right)$ and assume that $q \in Q$ where $Q$ is an admissible parameter space in which the constraints (3.3), smoothness criteria, and physical constraints on the parameters are satisfied. The finite dimensional parameter estimation problem is to then seek $\bar{q} \in Q$ which minimizes

$$
J^{N}(q)=\sum_{j}\left|\frac{\partial^{2} w^{\mathcal{N}}}{\partial t^{2}}\left(t_{j}, \hat{r}, \hat{\theta} ; q\right)-z_{j}\right|^{2}
$$

subject to $w^{\mathcal{N}}$ satisfying the approximate plate equations (hence the coefficients $\left\{w_{k}^{\mathcal{N}}\right\}$ of $w^{\mathcal{N}}$ must satisfy $(3.2))$. Here $z_{j}$ is an observation of acceleration taken at time $t_{j}$ and point $(\hat{r}, \hat{\theta})$ on the plate. Details regarding the convergence of the parameter estimates for general problems of this type can be found in [14].

In the results in the examples of the next section, minimization of the functional (3.4) was accomplished via a Levenberg-Marquardt routine with a stiff ODE solver being used to integrate the system (3.2) in order to obtain the model response at the sample points. This minimization can also be performed with various constrained optimization routines in which case, parameter constraints such as positivity can be enforced. 


\section{Experimental Results}

In the experimental results reported here, a circular aluminum plate with a single centered circular patch was considered (see Figure 3). The dimensions of the plate and piezoceramic patches are summarized in Table 1 . We note that the patch has a radius that is $\frac{1}{12}$ that of the plate and a thickness that is approximately $\frac{1}{7}$ of the plate thickness; hence it is quite small in relation to the plate. Table 1 also contains "handbook" values of the density, Young's modulus and Poisson ratio for the plate. We reiterate that while these values provide a starting point in the parameter estimation routine, they usually cannot be used in the final system model with any accuracy due to nonuniformities in the plate or boundary conditions, variations in materials, and the contributions due to the presence of the patches (this fact is illustrated in the examples).

To provide a basis for comparison between measured experimental natural frequencies and the analytic frequencies for a plate of this size to which no patches are bonded, analytic values were calculated using the plate dimensions and "handbook" parameter values summarized in Table 1. These analytic values are compiled in Table 2. In this latter table, $m$ refers to the Fourier number and $n$ denotes the order of the root to the Bessel functions which comprise the analytic solutions. Hence the analytic frequencies of the first four axisymmetric modes are $61.9,241.2,540.5$ and 959.5 hertz corresponding to $m=0, n=0,1,2$ and 3, respectively. The Fourier number $m$ can also be interpreted as the number of nodal diameters while $n$ is the number of nodal circles, not including the boundary. As will be seen in the examples, the experimental frequencies are, in many cases, significantly lower than the corresponding analytic values due to variations in material properties.

Time domain data was collected using accelerometers located at the points $A_{\ell}=\left(2^{\prime \prime}, 0\right), A_{c}=$ $(0,0)$ and $A_{r}=\left(2^{\prime \prime}, \pi\right)$ as depicted in Figure 3 (thus specifying $(\hat{r}, \hat{\theta})$ in $\left.(3.4)\right)$. This orientation of accelerometers permitted the collection of both axisymmetric and nonaxisymmetric data with the location chosen to avoid low-order nodal lines and circles. In all cases, data was obtained at a $12 \mathrm{KHz}$ sample rate so as to resolve any high frequency responses.

The experimental results reported here can be summarized as follows:

Example 1: Axisymmetric Excitation with Large Hammer - Open Circuit

Example 2: Axisymmetric Excitation with Large Hammer - Closed Circuit

Example 3: Axisymmetric Excitation with Small Hammer

Example 4: Axisymmetric Excitation through a Voltage Spike to Patch

Example 5: Nonaxisymmetric Excitation with Small Hammer

Note that both axisymmetric and general nonaxisymmetric responses are considered with input provided by various impact hammers as well as from the patches themselves. The damping effects due to the electric circuit containing the patch are also investigated. In each experiment, the goal is the estimation of the various physical parameters and the results from all experiments discussed here are summarized in Table 3. The consistency and/or variability of these estimates will be discussed in the examples presented below. 

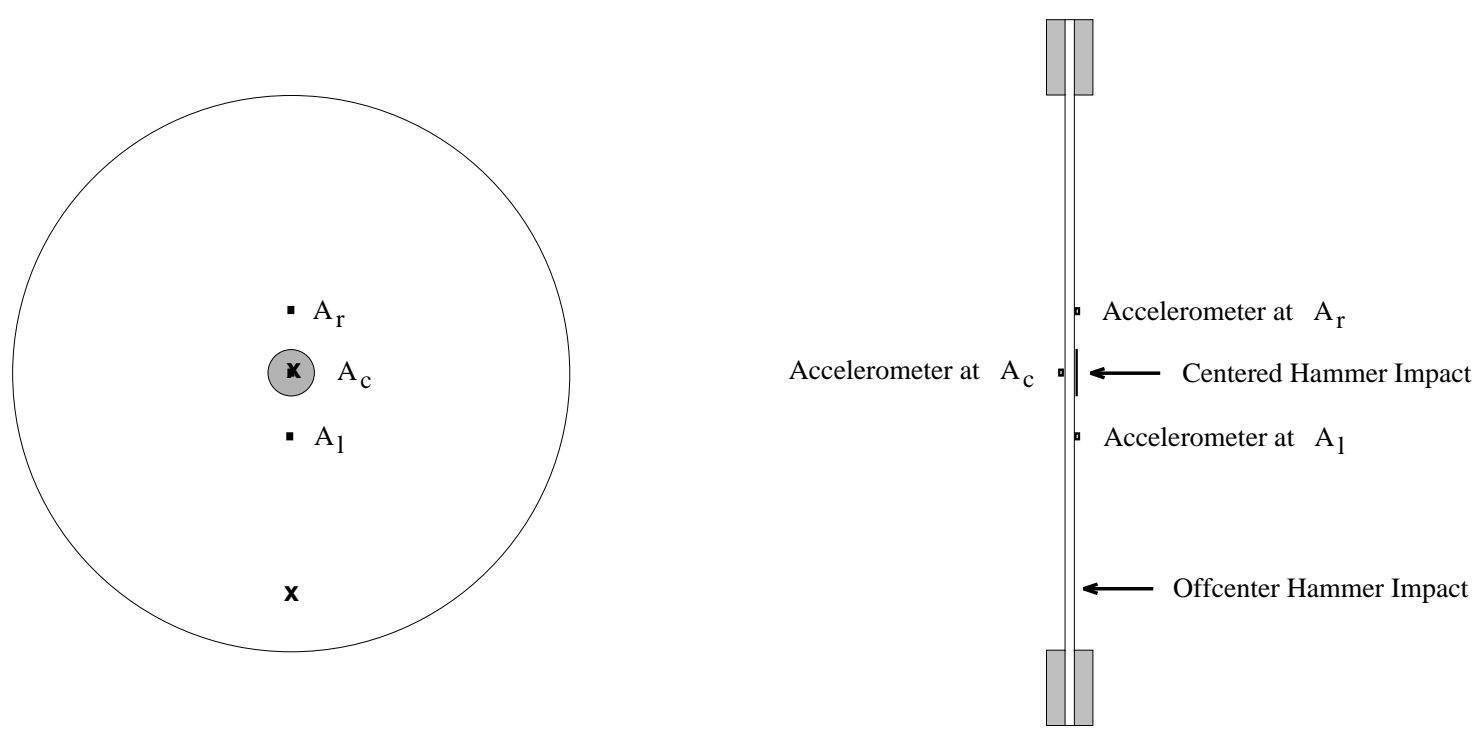

Figure 3. Clamped circular plate with a single centered piezoceramic patch. Accelerometers located at $A_{\ell}=\left(2^{\prime \prime}, 0\right), A_{c}=(0,0)$ and $A_{r}=\left(2^{\prime \prime}, \pi\right)$. Centered hammer impact at $(0,0)$, offcenter hammer impact at $\left(7 \cdot 27^{\prime \prime}, 0\right)$.

\begin{tabular}{l|l|l} 
& Plate Properties & Patch Properties \\
\hline Radius & $a=.2286 \mathrm{~m}\left(9^{\prime \prime}\right)$ & $\mathrm{rad}=.01905 \mathrm{~m}\left(.75^{\prime \prime}\right)$ \\
Thickness & $h=.00127 \mathrm{~m}\left(.05^{\prime \prime}\right)$ & $T=.0001778 \mathrm{~m}\left(.007^{\prime \prime}\right)$ \\
Young's Modulus & $E_{p}=7.1 \times 10^{10} \mathrm{~N} / \mathrm{m}^{2}$ & $E_{p e}=6.3 \times 10^{10} \mathrm{~N} / \mathrm{m}^{2}$ \\
Density & $\rho_{p}=2700 \mathrm{~kg} / \mathrm{m}^{3}$ & $\rho_{p e}=7600 \mathrm{~kg} / \mathrm{m}^{3}$ \\
Poisson ratio & $\nu_{p}=.33$ & $\nu_{p e}=.31$ \\
Strain Coefficient & & $d_{31}=190 \times 10^{-12} \mathrm{~m} / \mathrm{V}$ \\
\hline
\end{tabular}

Table 1. Dimensions and "handbook" characteristics of the plate and PZT piezoceramic patch.

\begin{tabular}{c|ccccccc}
\hline$n$ & $m=0$ & $m=1$ & $m=2$ & $m=3$ & $m=4$ & $m=5$ & $m=6$ \\
\hline 0 & 61.9 & 129.0 & 211.6 & 309.6 & 422.6 & 550.4 & 692.8 \\
1 & 241.2 & 368.9 & 513.0 & 673.3 & 849.8 & & \\
2 & 540.5 & 728.3 & 932.9 & & & & \\
3 & 959.5 & & & & & & \\
\hline
\end{tabular}

Table 2. Plate frequencies calculated using "handbook" dimensions and parameters under the assumption of a thin plate model with fixed boundary conditions. 


\begin{tabular}{c|c|c|ccccc}
\hline \multicolumn{2}{l|}{} & Analytic & Ex. 1 & Ex. 2 & Ex. 3 & Ex. 4 & Ex. 5 \\
\hline$\rho$ & Plate & 3.429 & 3.107 & 3.123 & 3.114 & 3.170 & 3.165 \\
$\left(\mathrm{~kg} / \mathrm{m}^{2}\right)$ & Plate + Pzt & & 3.131 & 3.230 & 2.993 & 3.216 & 3.179 \\
\hline$D$ & Plate & 13.601 & 11.310 & 11.270 & 11.205 & 11.151 & 11.361 \\
$(N m)$ & Plate + Pzt & & 11.381 & 11.302 & 11.674 & 11.506 & 11.783 \\
\hline$c_{D}$ & Plate & & $1.161-4$ & $1.443-4$ & $9.358-6$ & $2.816-5$ & $2.598-5$ \\
$(N m s e c)$ & Plate + Pzt & & $1.290-4$ & $2.031-4$ & $9.392-6$ & $3.211-5$ & $2.693-5$ \\
\hline$\nu$ & Plate & .33 & .331 & .331 & .330 & .326 & .330 \\
& Plate + Pzt & & .326 & .325 & .327 & .325 & .328 \\
\hline$\gamma\left(s e c N / m^{3}\right)$ & & 11.57 & 17.02 & 58.57 & 58.97 & 45.71 \\
\hline \hline \multicolumn{2}{c|}{$\mathcal{K}^{B}(N / V)$} & & & & & .006074 & \\
\hline
\end{tabular}

Table 3. Analytic and experimental parameters values obtained in Examples 1 - 5.

\section{Example 1: Axisymmetric Excitation with Large Hammer - Open Circuit}

In the first set of experiments, the plate was excited through an impulse delivered by a large impact hammer having a plastic tip (the force transducer on the hammer delivered $50 \mathrm{mV} / \mathrm{lb}$ ). The impact was delivered to the center of the plate and data was collected from accelerometers located at the points $A_{\ell}=\left(2^{\prime \prime}, 0\right), A_{c}=(0,0)$ and $A_{r}=\left(2^{\prime \prime}, \pi\right)$ (see Figure 3$)$. The excitation of the structure in this manner provided a primarily axisymmetric response with the purely axisymmetric component being measured by the centered accelerometer. Data obtained from off-center accelerometers indicated that while slight nonaxisymmetric vibrations were present, their effect was minimal.

During the collection of this data, the circuit involving the piezoceramic patch was left open to minimize piezoelectric effects due to the bending patch (with a closed circuit, the voltage produced when the patch vibrates is fed back to the patch which in turn produces a bending moment; the damping and stiffening effects which occur in this case are investigated in the next example).

Minimization of the function (3.4) was performed using the time history of the acceleration obtained from the centered accelerometer (at $A_{c}=(0,0)$ ). For this experiment, fixed-edge boundary conditions were assumed and hence the optimization was performed subject to $w^{\mathcal{N}}$ satisfying the discretization of (2.7). The estimated parameters $\rho, \gamma, D, \nu$ and $c_{D}\left(\mathcal{K}^{B}\right.$ was not estimated here since there is no patch input) are recorded in Table 3 while model-based results obtained with these values are plotted against experimental results in Figures 4 and 5 . We reiterate that in these plots, both the data and calculated model response were obtained at the center point of the plate.

As indicated by the frequency results in Figure 5, four axisymmetric modes, having frequencies of 59.3, 227.8,516.4 and 917.7, were excited in this experiment. The results in both figures demonstrate that the parameter estimates in Table 3 lead to a very close matching of the first two frequencies. The overdamping of the higher frequency modes is characteristic of the Kelvin-Voigt damping model and this leads to the very slight variation seen in the time history when comparing the experimental data and model response. 
To demonstrate the distributed nature of the model, the parameters obtained using data from the centered accelerometer, as summarized in Table 3, were used to calculate the model response at the offcenter point $A_{r}=\left(2^{\prime \prime}, \pi\right)$. The results are plotted along with the experimental data at that point in Figures 6 and 7. From the frequency results in Figure 7, it can be seen that the primary response at that point is in the first two axisymmetric modes, and while the model response in the first mode is slightly larger than the corresponding experimental result, the agreement is very close in light of the fact that experimental data from this accelerometer was not used when determining the physical parameters. Similar results were found at the point $A_{\ell}=\left(2^{\prime \prime}, 0\right)$, thus demonstrating the distributed nature of the model.

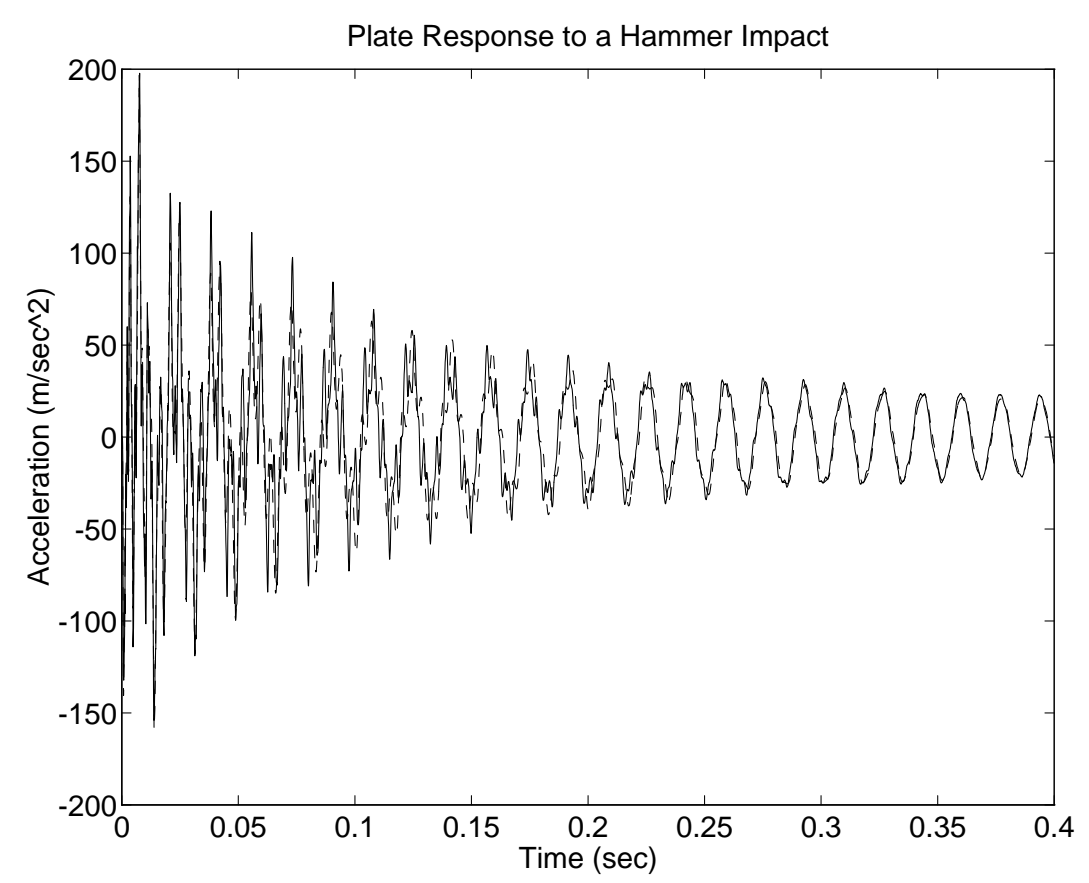

Figure 4. Time history of the Experiment 1 data measured at $A_{c}=(0,0)$ and model response with estimated parameters, _- (Experimental Data), - - (Model Response). 


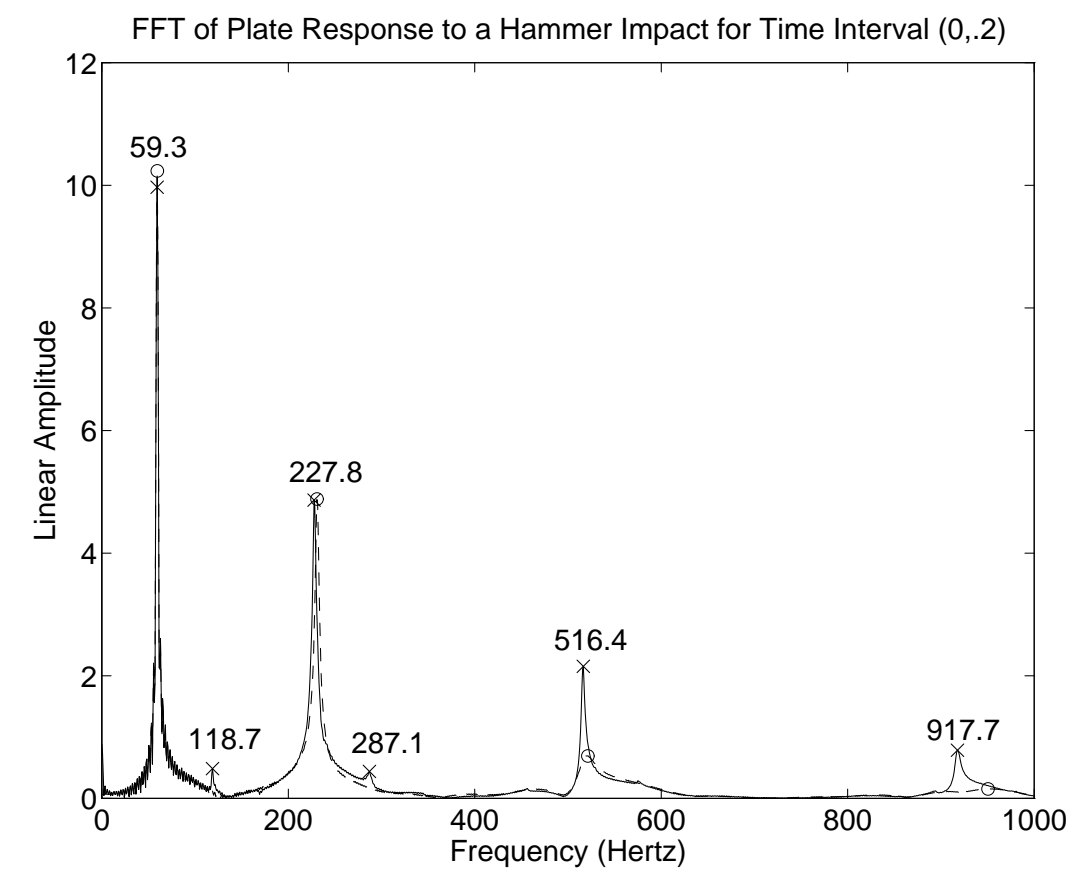

Figure 5. Frequency content of the Experiment 1 data measured at $A_{c}=(0,0)$ and thin plate model with estimated parameters, $\mathrm{x}-($ Experimental Data), o - - - (Model Response).

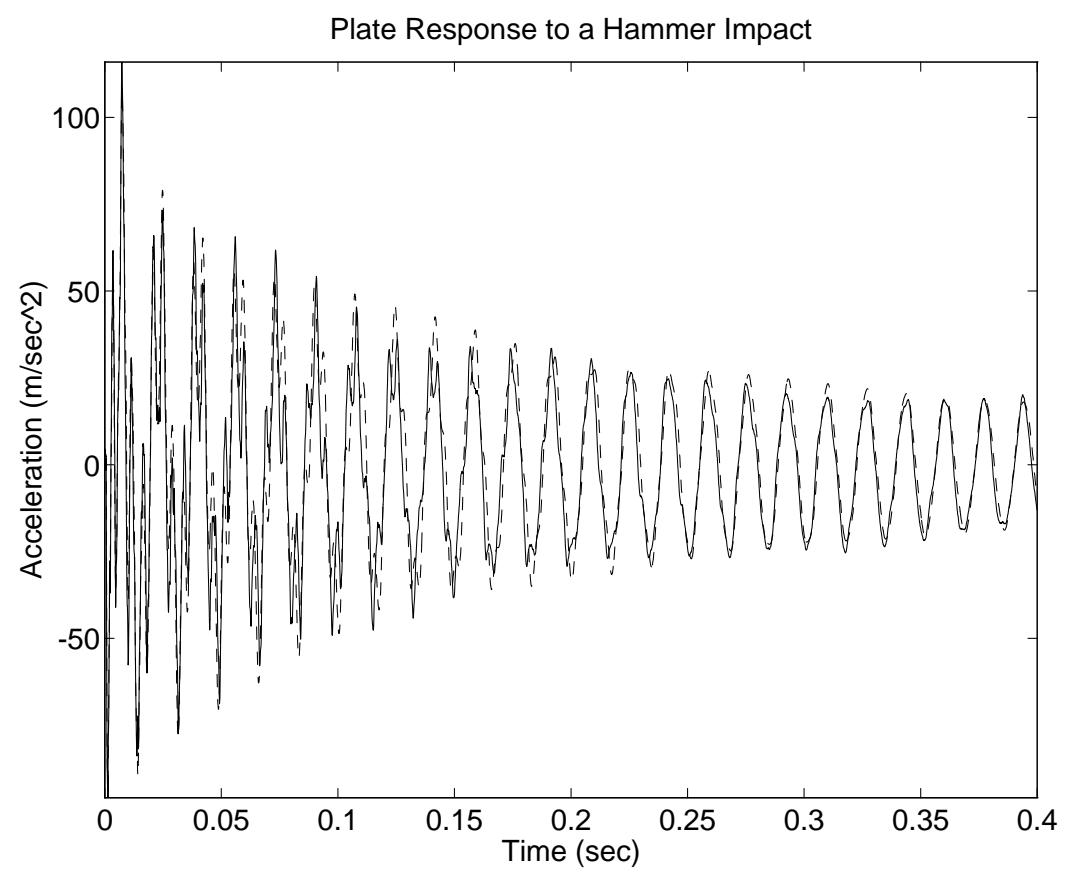

Figure 6. Time history of the Experiment 1 data measured at $A_{r}=(2, \pi)$ and model response with estimated parameters, - (Experimental Data), - - (Model Response). 


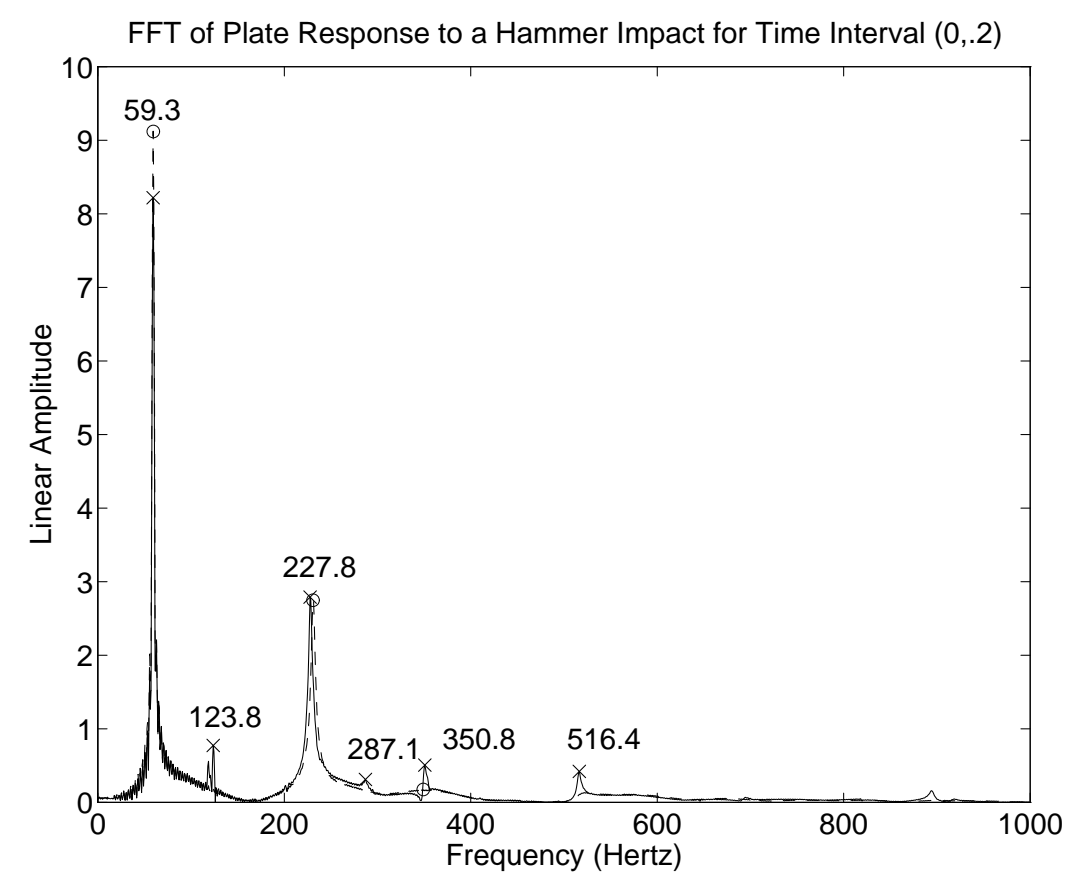

Figure 7. Frequency content of the Experiment 1 data measured at $A_{r}=(2, \pi)$ and thin plate model with estimated parameters, $\mathrm{x}-($ Experimental Data), o - - - (Model Response).

\section{Example 2: Axisymmetric Excitation with Large Hammer - Closed Circuit}

As discussed previously, the piezoelectric effect is manifested in two ways in the patches. In one case, vibrations in the plate and hence the patch lead to generated strains which in turn produce voltages, whereas the converse effect leads to generated strains in response to an input voltage. The completion of the circuit involving the piezoceramic patch leads to a strong interaction between these effects, and indeed, the shunting of the patch by simply connecting the leads is a recognized means of increasing system damping and changing stiffness properties [16].

In this example, the effects of closing the circuit on the estimated parameters are investigated. The experimental setup is identical to that described in the previous example except that in this case, the circuit involving the piezoceramic patch was closed. For experiments in which input to a piezoceramic actuator is used to control the system, this is a more realistic scenario since the circuits must be complete in any control setup. As in the previous example, an impact hammer hit to the plate center was used to obtain an axisymmetric response and data was obtained from accelerometers located at the points $A_{c}, A_{r}$ and $A_{\ell}$ depicted in Figure 3.

To obtain model responses for this case, three sets of parameters, as summarized in Table 4, were used. The first set of parameters was obtained by minimizing the functional (3.4) using data from the centered accelerometer. These parameters can be compared with those in the second set which were obtained in the first experiment. To obtain the third set, the analytic values for the density, flexural rigidity and Poisson ratio for the plate were used throughout the 
structure while $\gamma$ and $c_{D}$ from the first data set were used as damping values. The use of the third data set simulates the results that are obtained if one simply uses "handbook" values for the density, flexural rigidity and Poisson ratio.

As demonstrated by the time history and corresponding frequency plots in Figure 8a, results comparable to those obtained in Example 1 can be obtained when the physical parameters are obtained using fit-to-data techniques. By comparing the parameters obtained here with those of the first experiment, however, one sees some variation due to the circuit effects on the piezoceramic patches. The most marked difference is an increase in damping which results when the system is closed. Since the damping effects due to the circuit are not included in the model, the optimization routine increased the viscous damping coefficient $\gamma$ and Kelvin-Voigt parameter $c_{D}$. As noted in the plots of Figure 8a, this compensation for the damping leads to a good model fit to the data even though the mechanism for the unmodeled circuit damping differs from the internal and viscous damping included in the model. While some difference in density and stiffness also occur, these effects are less pronounced due to the small size of the patch in relation to the plate.

The experimental data and model response obtained with parameters from Experiment 1 (open circuit) are plotted in Figure 8b. As noted in these plots, the model response is significantly underdamped since the effects of damping due to the closed circuit were not considered in Example 1. Moreover, a slight shift in frequency due to changes in $\rho, D$ and $\nu$ can also be noted. This illustrates some of the variations which result from changing the configuration of the electric circuit and highlights the fact that identification procedures should be performed in the setting in which applications or control are to be considered.

Finally, the experimental data and model response obtained with the third set of parameters (analytic values of $\rho, D$ and $\nu$ ) are plotted in Figure $8 \mathrm{c}$. As noted in both time domain and frequency plots, the frequency of the model response is much too large in this case, namely due to the fact that the analytic value of the flexural rigidity is approximately $17 \%$ larger than the estimated values. This illustrates the fact that even those parameters for which "handbook" values exist must be estimated through parameter identification techniques in order to guarantee an accurate model.

\begin{tabular}{c|c|c|c|c}
\hline \multicolumn{2}{l|}{} & $\begin{array}{c}\text { Estimated } \\
\text { Parameters }\end{array}$ & $\begin{array}{c}\text { Example 1 } \\
\text { Parameters }\end{array}$ & $\begin{array}{c}\text { Analytical } \\
\text { Parameters }\end{array}$ \\
\hline $\begin{array}{c}\rho \cdot \text { thickness } \\
\left(\mathrm{kg} / \mathrm{m}^{2}\right)\end{array}$ & Plate & 3.123 & 3.107 & 3.429 \\
Plate + Pzt & 3.230 & 3.131 & 3.429 \\
\hline$D$ & Plate & 11.270 & 11.310 & 13.601 \\
$(N \cdot m)$ & Plate + Pzt & 11.302 & 11.381 & 13.601 \\
\hline$c_{D}$ & Plate & $1.443-4$ & $1.161-4$ & $1.161-4$ \\
$(N \cdot m \cdot s e c)$ & Plate + Pzt & $2.031-4$ & $1.290-4$ & $1.290-4$ \\
\hline$\nu$ & Plate & .331 & .331 & .330 \\
& Plate + Pzt & .325 & .326 & .330 \\
\hline \multicolumn{2}{r|}{$\gamma(s e c \cdot N / m)$} & 17.021 & 11.569 & 11.569 \\
\hline
\end{tabular}

Table 4. Analytic and experimental values of the physical parameters. The estimated parameters in Column 3 were obtained using Experiment 2 data. 

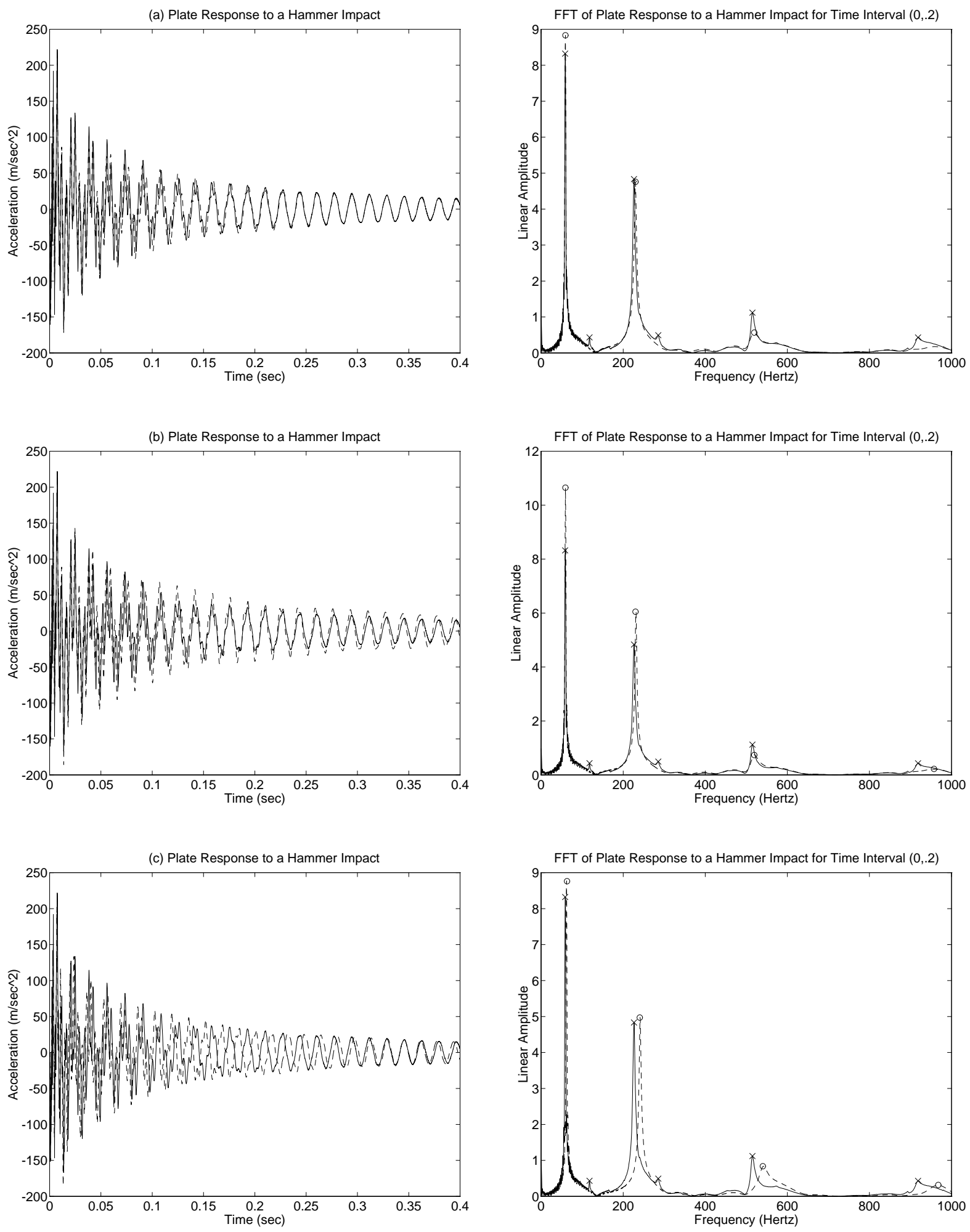

Figure 8. Experiment 2 data measured at $A_{c}=(0,0)$ and model response with (a) estimated parameters, (b) parameters from Example 1, and (c) analytic parameters; $\mathrm{x}-$ (Experimental Data), o--- (Model Response). 


\section{Example 3: Axisymmetric Excitation with Small Hammer}

In the previous two experiments, the plate was excited through impacts from a large hammer having a soft tip. This resulted in the excitation of four axisymmetric modes having frequencies ranging from approximately $60 \mathrm{~Hz}$ to $920 \mathrm{~Hz}$. To investigate the suitability of the model when a wider range of frequencies are excited, a small impact hammer (with a force transducer delivering $100 \mathrm{mV} / \mathrm{lb}$ ) having a metal tip was also used with the results being reported in this example.

As in the previous example, a centered hit was used to evoke an axisymmetric response with data being obtained from accelerometers located at $A_{c}, A_{\ell}$ and $A_{r}$. The leads to the piezoceramic patches were left disconnected, thus minimizing the damping effects due to the circuit and patch. The minimization of the functional (3.4) was performed with data from the centered accelerometer and the resulting estimated parameters are summarized in Table 3 . The model response and experimental data from the centered accelerometer are plotted in Figure 9 and 10. As indicated by the frequency plots in Figure 10, six modes were accurately matched with these estimated parameter values with expected overdamping of the high frequency 2814 and $3661 \mathrm{~Hz}$ modes.

In comparing the parameter estimates of Examples 1 and 2 in Table 3 , it can be seen that while little change occurs in $\rho, D$ and $\nu$, there is some variation in the viscous damping constant $\gamma$ and the internal Kelvin-Voigt parameter $c_{D}$. This is due to the different frequency responses in the two experiments and again reflects some limitations in the damping model. When the large hammer was used to excite the plate, the primary response was in the lower frequency modes and the parameters obtained from the minimization of (3.4) yielded a model which matched the lower frequencies but overdamped the higher frequencies having less energy. The use of the small hammer with a metal head resulted in data in which the primary response was in the $918 \mathrm{~Hz}$ mode with very little energy in the $60 \mathrm{~Hz}$ mode. This shift in the excited frequencies generally leads to a reduction in the estimated values of $c_{D}$ and an increase in $\gamma$ (see also Examples 4 and 5). 


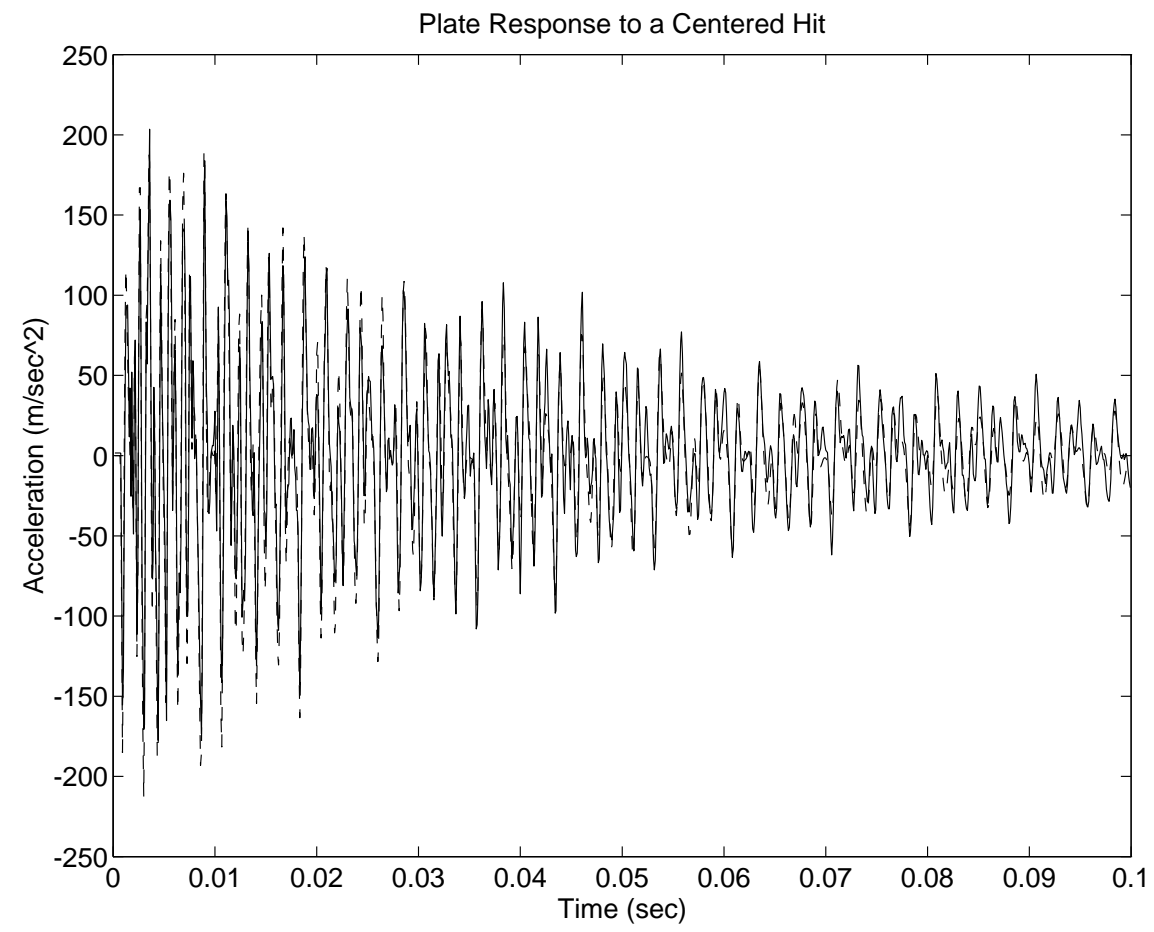

Figure 9. Time history of the Experiment 3 data measured at $A_{c}=(0,0)$ and model response with estimated parameters, - (Experimental Data), - - (Model Response).

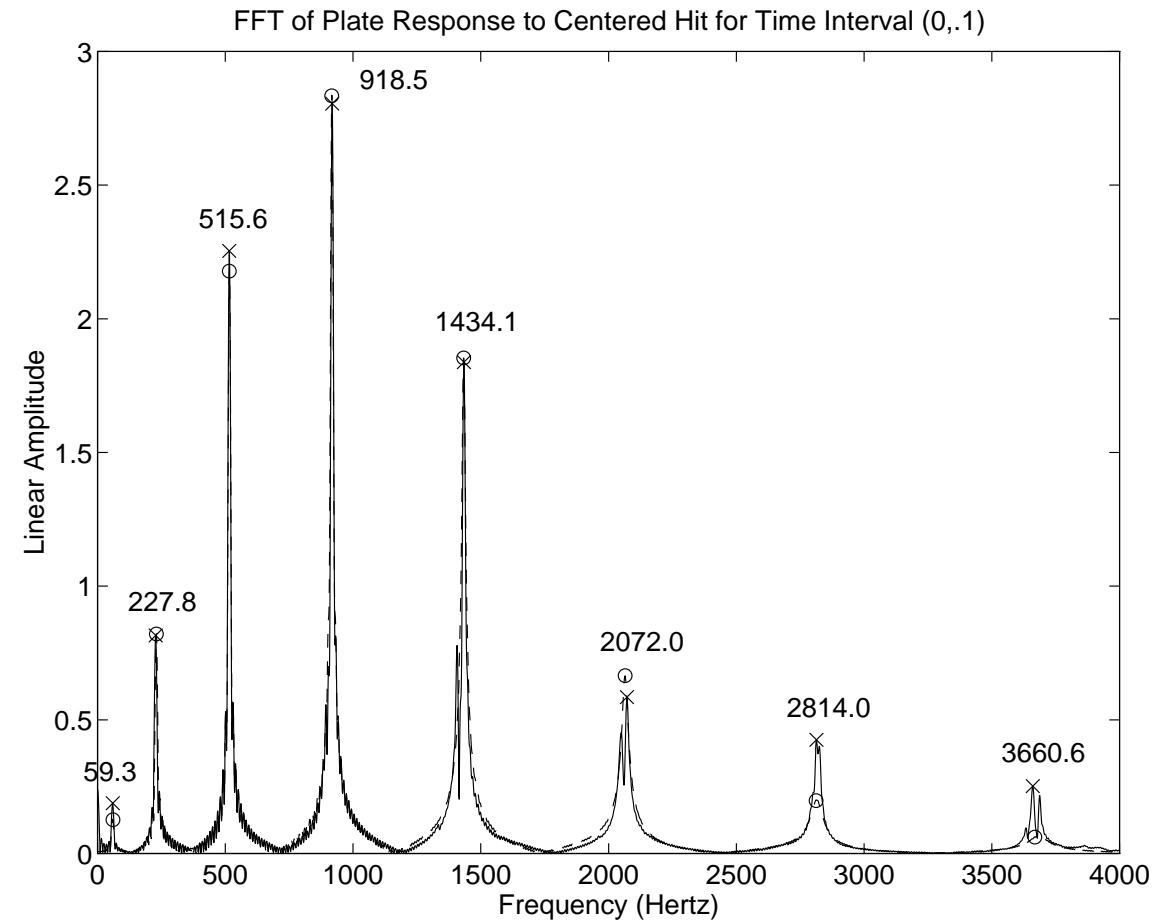

Figure 10. Frequency content of the Experiment 3 data measured at $A_{c}=(0,0)$ and thin plate model with estimated parameters, $\mathrm{x}-($ Experimental Data $), \mathrm{o}_{-}--$(Model Response). 


\section{Example 4: Axisymmetric Excitation - Voltage Spike to Patch}

A second means of exciting the plate is through a voltage spike to the piezoceramic patch and results obtained in that manner are reported here. Because the active patch was centered on the plate, this yielded an axisymmetric response and data from the centered accelerometer was used when minimizing the functional (3.4).

The estimated physical parameters $\rho, D, \nu, c_{D}$ and $\gamma$ as well as the patch input parameter $\mathcal{K}^{B}$ are summarized in Table 3 and the resulting model response is plotted along with the experimental data from the centered accelerometer in Figure 11 and 12. As indicated by the time and frequency plots in the figures, the plate response obtained in this manner is quite similar to that obtained by exciting the plate with the small metal-tipped hammer. In comparing the estimated parameters from Examples 3 and 4, it is noted that there is very little variation in either $\rho, D, \nu$ or $c_{D}, \gamma$, in spite of the differing mechanisms for exciting the system.

The estimated value .006074 for the patch parameter $\mathcal{K}^{B}$ is seen to be approximately $48 \%$ of the value .0126 predicted by the model (2.5) with the values of $E_{p e}, \nu_{p e}, h, T$ and $d_{31}$ specified in Table 1 and $T_{b e}$ taken to be 0 . Some of this variation can be attributed to patch material values which differ slightly from those summarized in Table 1 . While differences occur between the "handbook" values of the Young's modulus and Poisson ratio and the "true" parameters for the experimental patch, perhaps the biggest source of variation occurs in the values for the strain constant $d_{31}$. The value reported in the product literature (and given in Table 1) was obtained through static tests while the estimated value is obtained in a dynamic setting which tends to decrease the realized values of strain parameters such as $d_{31}[15,19,20]$. Hence while the analytic values given by the model (2.5) can be used as starting values in the optimization routine, they will not in general yield an accurate model response due to physical variations in the material patch properties.

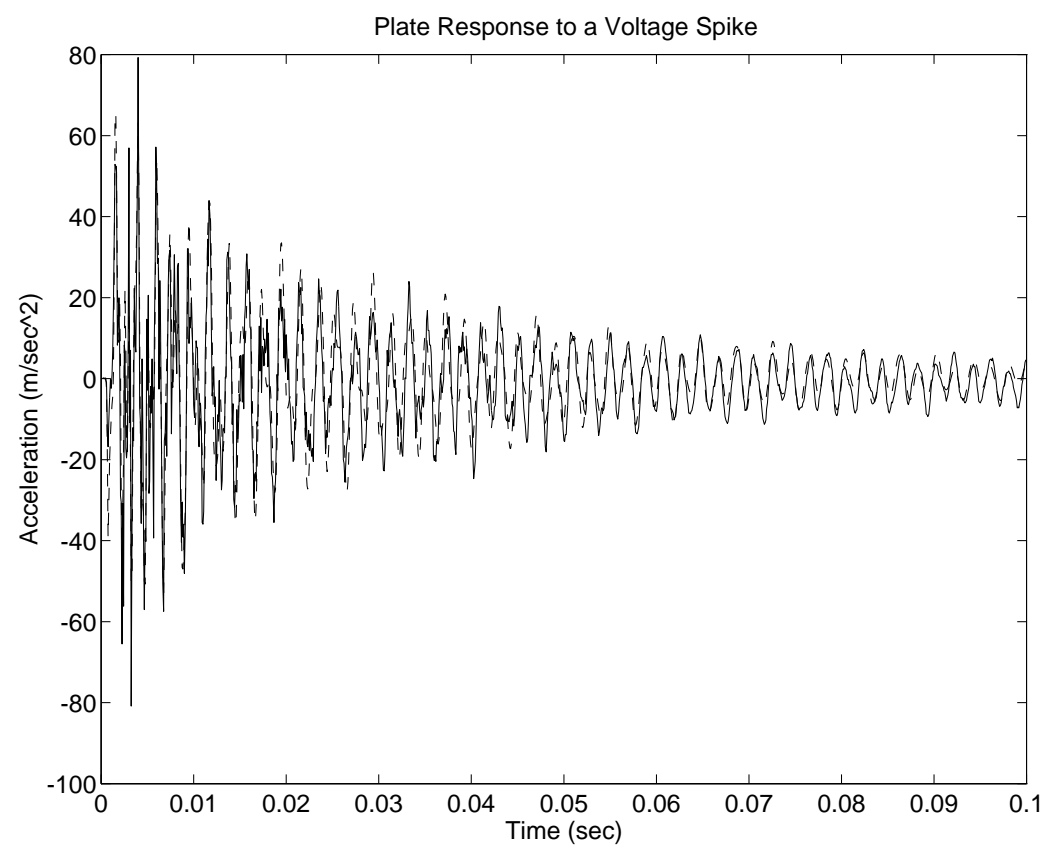

Figure 11. Time history of the Experiment 4 data measured at $A_{c}=(0,0)$ and model response with estimated parameters, (Experimental Data), - - (Model Response). 


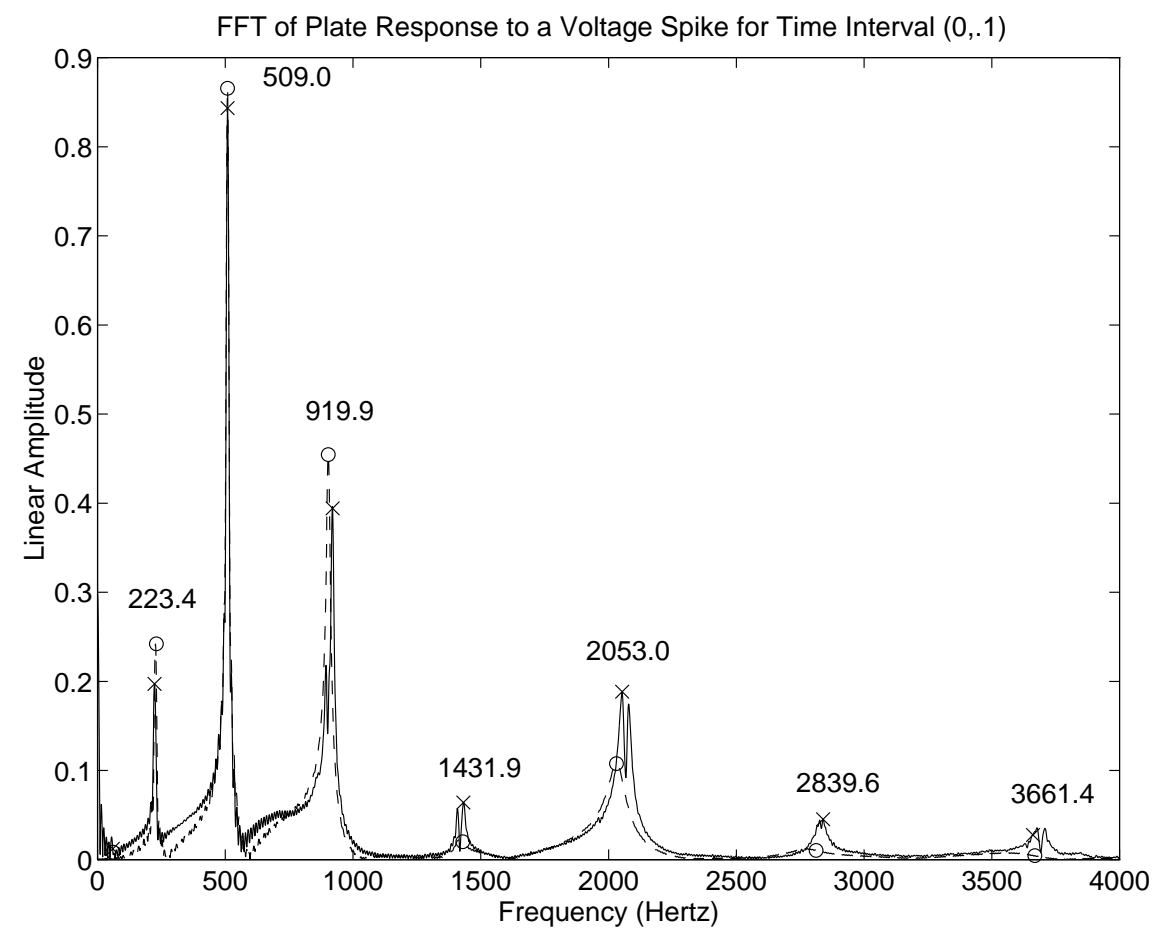

Figure 12. Frequency content of the Experiment 4 data measured at $A_{c}=(0,0)$ and thin plate model with estimated parameters, $\mathrm{x}-($ Experimental Data $), \mathrm{o}_{-}--$(Model Response).

\section{Example 5: Nonaxisymmetric Excitation with Small Hammer}

In this experiment, a nonaxisymmetric response was obtained through a small hammer impact at the point $\left(7.27^{\prime \prime}, 0\right)$ (see Figure 3 ). The leads to the piezoceramic patch were disconnected in this experiment to minimize damping effects due to the circuit and piezoelectric properties of the patch. Data was again measured via the three accelerometers located at $A_{r}=\left(2^{\prime \prime}, 0\right), A_{c}=(0,0)$ and $A_{\ell}=\left(2^{\prime \prime}, \pi\right)$. Optimization was performed using the data from the accelerometer located at $A_{r}=\left(2^{\prime \prime}, 0\right)$ and the estimated parameters values are summarized in Table 3.

Time and frequency plots of the experimental data from the right $\left(A_{r}\right)$, centered $\left(A_{c}\right)$ and left $\left(A_{\ell}\right)$ accelerometers as well as corresponding model responses are given in Figure $13 \mathrm{a}, \mathrm{b}$ and $c$, respectively. The observed experimental frequencies as well as the calculated model frequencies at the three accelerometers are tabulated in Table 5.

From the frequency plots in figure $13 \mathrm{a}$, it can be seen that the model very accurately matches the $(n, m)=(0,0),(0,2),(0,3),(1,1),(1,2),(2,0),(2,1)$ and $(0,4)$ modes while significantly underdamping the $(1,0)$ and $(0,1)$ modes (see Table 2 to compare the observed frequencies with the corresponding modes). As expected, the higher-order modes are overdamped as is typical with the Kelvin-Voigt damping mechanism.

Similar results are observed in the plots in Figure 13c which depict the acceleration data and model response at $A_{\ell}$ (recall that the data from the right accelerometer was used for obtaining the parameters). In addition to the previously matched modes, this data contains a stronger 
response in the $(0,4)$ mode $(408 \mathrm{~Hz})$ which is accurately matched by the model. Although the $(1,0)$ and $(0,1)$ modes are still underdamped, the accurate matching of 9 modes demonstrates the distributed nature of this model.

The underdamping of the $(1,0)$ mode is very evident in both the time domain and frequency plots of the data and model response at the centered accelerometer (Figure 13b). By comparing the relative degree of underdamping that is observed at $A_{c}$ with that seen at $A_{r}$ or $A_{\ell}$, it can be seen that the results are comparable. However, the different distribution of energy in the axisymmetric and nonaxisymmetric modes leads to larger discrepancies between the model and experimental data measured at $A_{c}$ than were observed at the noncentered points. The underdamping of the $(1,0)$ and $(0,1)$ modes again illustrates some of the limitations of the damping model being used in these investigations.

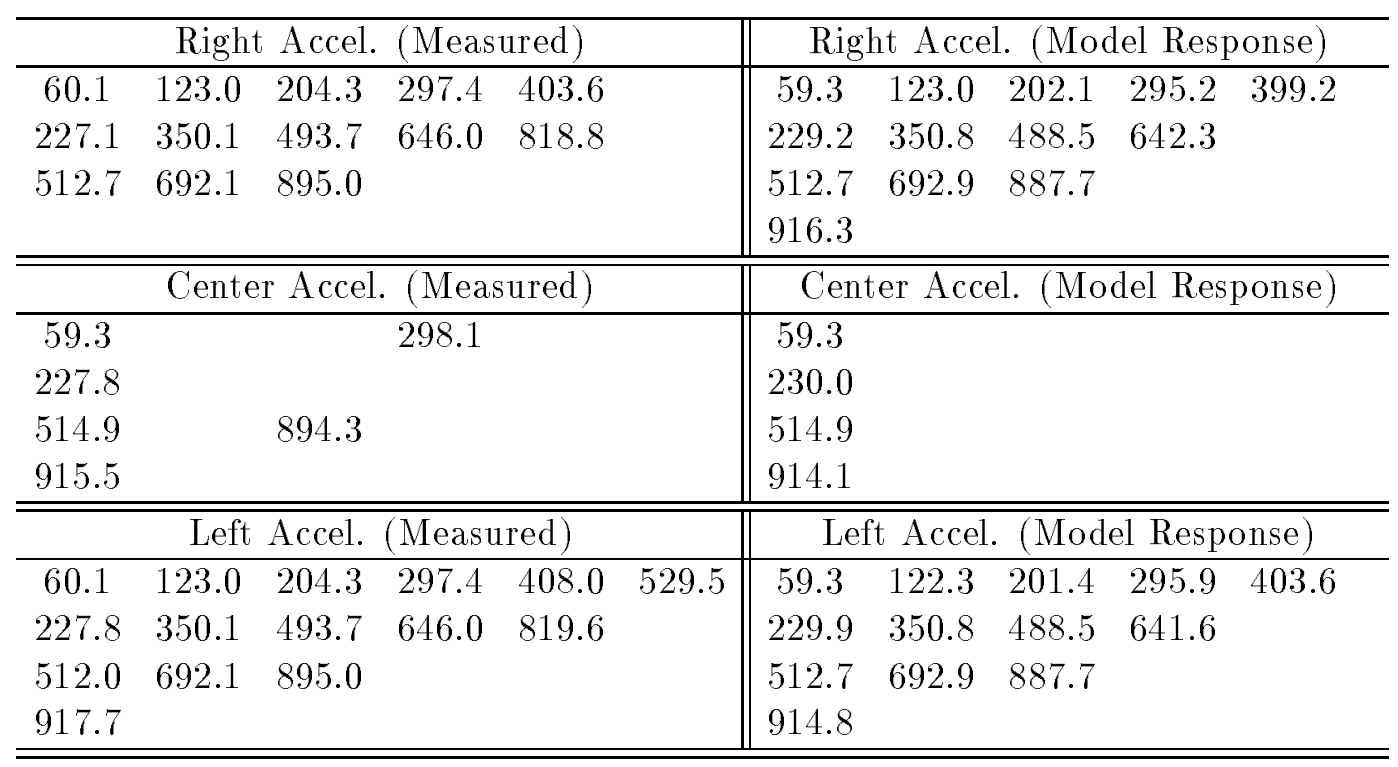

Table 5: Observed frequencies in the Experiment 5 data and model response. 

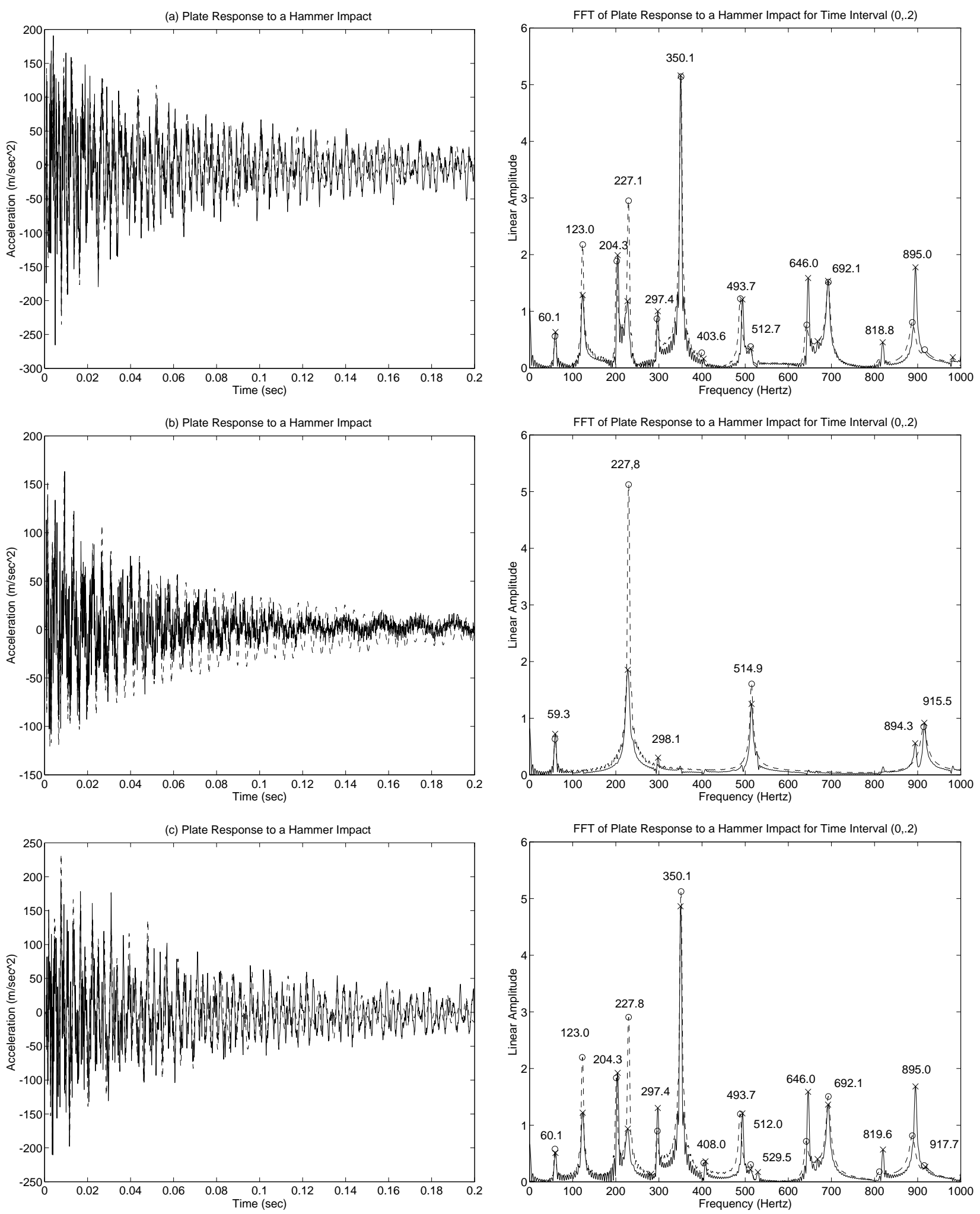

Figure 13. Experiment 5 data and model response at (a) $A_{r}=\left(2^{\prime \prime}, 0\right)$, (b) $A_{c}=(0,0)$ and (c) $A_{\ell}=\left(2^{\prime \prime}, \pi\right) ; \mathrm{x}-($ Experimental Data $), \mathrm{o}_{---}$(Model Response). 


\section{Conclusion}

In this paper, we have considered issues associated with the estimation of parameters in a PDE-based model for a vibrating plate. Specifically, we considered a clamped thin circular plate with surface-mounted piezoceramic patches. Thin plate equations which accounted for both the passive and active contributions from the patches were used to model the dynamics of the system. The unknown parameters in the model included structural parameters (density, flexural rigidity, Poisson ratio and material and air damping) and patch parameters. The structural parameters were taken to be piecewise constant in order to account for the presence and differing material properties of the patches. It should be noted that all modeling equations for the system were derived using Newtonian principles (force and moment balancing), and all parameters represent physical quantities in the system.

When designing and performing experiments, two issues were considered. The first concerned the ability of the PDE model to accurately and consistently describe the physics of the system under a variety of inputs and responses. Secondly, it is well-known that closing or shunting the circuit containing the piezoceramic patch provides additional damping, and this was investigated in the context of the PDE model.

With regards to the first issue, experiments were performed in which the plate was excited with a variety of inputs (including impact hammers and voltage spikes to the patches) which excited from four to fifteen frequencies ranging from $60 \mathrm{~Hz}$ to $4000 \mathrm{~Hz}$. The matching of up to six axisymmetric and eight nonaxisymmetric frequencies illustrated that the thin plate model was appropriate and sufficiently accurate for the experimental plate under consideration. Moreover, the distributed nature of the PDE model means that it accurately describes the physics of the entire plate including points not used in the optimization process. As demonstrated by results reported in [1], the accuracy of the model, with parameters estimated in the manner discussed here, contributed to the good vibration attenuation attained when the model was incorporated in a PDE-based controller.

When comparing the parameters estimated in the various experiments, it was noted that the density, flexural rigidity and Poisson ratios were consistent across all experiments. There was some variation in the damping parameters depending on the frequency content of the data. In experiments with minimal low frequency excitation but substantial energy in the high frequencies, the Kelvin-Voigt damping coefficient $c_{D}$ was smaller and air damping $\gamma$ higher than in experiments in which the response was dominated by the primary mode. This indicates the necessity of estimating parameters with a response in the frequency range under consideration and illustrates a limitation in the damping model.

The damping which results when the circuit involving the piezoceramic patch is closed was investigated by performing a series of experiments with open and closed circuits. The estimated parameters and model responses for the two cases were then compared. As expected, the plate response with the closed circuit was more highly damped than that obtained with the open circuit, and the optimization routine compensated by increasing the material damping coefficients. While the damping provided by the circuit is not directly modeled by the Kelvin-Voigt or viscous damping terms, it does produce an effect in the system which is phenomenologically similar to Kelvin-Voigt and viscous damping, and hence accurate model fits were obtained with the estimated parameters. We emphasize that if the applications of interest involve such a

closed circuit, parameter estimation should be performed in this regime so as to account for 
the additional damping.

We reiterate that while the fixed-edge boundary conditions (2.6) adequately modeled the boundary dynamics for the setup under consideration, in many cases, energy loss through the boundary clamps will make the fixed-edge model inadequate. In such cases, an "almost fixed" boundary moment model of the type discussed in [9] may provide a more accurate description of edge physics. Experimental results pertaining to the use of that model for describing the plate dynamics when boundary clamps are loosened can be found in [2].

Finally, while the investigations here pertained to a circular plate, the issues are important in a large number of applications involving vibrating structures, and the specific results reported here may indicate directions to be followed when developing and applying PDE models to more complex structures. As indicated by parameter estimation results reported here and control results reported in [1], the use of PDE models can lead to accurate descriptions of structural systems (even in the presence of actuators and sensors) which can then be successfully incorporated in PDE-based controllers.

\section{Acknowledgements}

The authors thank Dr. Yun Wang, Brooks Air Force Base, for advice offered during numerous conversations regarding the parameter estimation techniques used in this investigation.

\section{References}

[1] H.T. Banks, D.E. Brown, V. Metcalf, R.J. Silcox, R.C. Smith, "Experimental Confirmation of a PDE-Based Approach to Design of Feedback Controls," SIAM Journal of Optimization and Control, submitted, 1995.

[2] H.T. Banks, D.E. Brown, R.J. Silcox, R.C. Smith and Y. Wang, "Modeling and Estimation of Boundary Parameters for Imperfectly Clamped Structures," to be submitted as an IC ASE report.

[3] H.T. Banks, D. Cioranescu and D.A. Rebnord, "Homogenization Models for 2-D Grid Structures," Center for Research in Scientific Computation Technical Report CRSC-TR924, June 1992, N.C. State Univ., J. Asymptotic Analysis, to appear.

[4] H.T. Banks and D.A. Rebnord, "Estimation of Material Parameters for Grid Structures," J. Math. Systems, Estimation and Control, 1, pp. 107-130, 1991.

[5] H.T. Banks and D.A. Rebnord, "Analytic Semigroups: Applications to Inverse Problems for Flexible Structures," Differential Equations with Applications, (Intl. Conf. Proc., Retzhof, Austria), Marcel Dekkar, pp. 21-35, 1991.

[6] H.T. Banks and R.C. Smith, "Well-Posedness of a Model for Structural Acoustic Coupling in a Cavity Enclosed by a Thin Cylindrical Shell," ICASE Report 93-10, Journal of Mathematical Analysis and Applications, 191, pp. 1-25, 1995. 
[7] H.T. Banks and R.C. Smith, "The Modeling and Approximation of a Structural Acoustics Problem in a Hard-Walled Cylindrical Domain," Center for Research in Scientific Computation Technical Report CRSC-TR94-26, December 1994, N.C. State Univ., in Dynamics and Control of Distributed Systems, Editors: H.S. Tzou and L.A. Bergman, Cambridge University Press, to appear.

[8] H.T. Banks, R.C. Smith and Y. Wang, "The Modeling of Piezoceramic Patch Interactions with Shells, Plates and Beams," Quarterly of Applied Mathematics, 53(2), 1995, 353-381.

[9] H.T. Banks, R.C. Smith and Y. Wang, "Modeling and Parameter Estimation for an Imperfectly Clamped Plate," Center for Research in Scientific Computation Technical Report, CRSC-TR95-2, Computation and Control IV, Proc. Fourth Bozeman Conf., Bozeman, MT, 1994, Progress in Systems and Control Theory, Birkhäuser Boston, Inc., 1995, to appear.

[10] H.T. Banks, R.C. Smith and Y. Wang, "Parameter Estimation for an Imperfectly Clamped Plate - Numerical Examples," Proceedings of the $15^{\text {th }}$ ASME Biennial Conference on Vibration and Noise, Sept. 17-21, 1995.

[11] H.T. Banks, Y. Wang and D.J. Inman, "Bending and Shear Damping Damping in Beams: Frequency Domain Estimation Techniques," ASME J. Vibration and Acoustics, 116, pp. 188-197, 1994.

[12] H.T. Banks, Y. Wang, D.J. Inman and H. Cudney, "Parameter Identification Techniques for the Estimation of Damping in Flexible Structure Experiments," Proc. $26^{\text {th }}$ IEEE Conf. on Dec. and Control, December 1987, Los Angeles, pp. 1392-1395.

[13] H.T. Banks, Y. Wang, D.J. Inman and J.C. Slater, "Variable Coefficient Distributed Parameter System Models for Systems with Piezoceramic Actuators and Sensors," Center for Research in Scientific Computation Technical Report CRSC-TR92-9, September 1992, N.C. State Univ., Proc. $31^{\text {st }}$ IEEE Conf. on Dec. and Control, Tucson, Dec. 1992, pp. 1803-1808.

[14] H.T. Banks, Y. Wang, D.J. Inman and J.C. Slater, "Approximation and Parameter Identification for Damped Second Order Systems with Unbounded Input operators," Center for Research in Scientific Computation Technical Report CRSC-TR93-9, May 1993, N.C. State Univ., Control: Theory and Advanced Technology, 10, pp. 873-892, 1994.

[15] EDO Acoustics Corporation Division, 2645 South 300 West, Salt Lake City, UT 84115, (801) 486-2115, personal communications.

[16] N.W. Hagood and A. von Flotow, "Damping of Structural Vibrations with Piezoelectric Materials and Passive Electrical Networks," Journal of Sound and Vibration, 146(2), pp. 243-268, 1991.

[17] C.M. LaPeter, “Application of Distributed Measurements for Finite Elements Model Verification," Master's Thesis, Virginia Polytechnic Institute and State University, May 1992. 
[18] K.D. Murphy, L.N. Virgin and S.A. Rizzi, "Free Vibration of Thermally Loaded Panels Including Initial Imperfections and Post-Buckling Effects," NASA Technical Memorandum 109097, April 1994.

[19] A.B. Palazzollo, S. Jagannathan, A.F. Kascak, T. Griffin, J. Giriunas, G.T. Montague, "Piezoelectric Actuator-Active Vibration Control of the Shaft Line for a Gas Turbine Engine Test Stand," proceedings of the International Gas Turbine and Aeroengine Congress and Exposition, Cincinnati, OH, May 24-27, 1993, ASME Paper 93-GT-262.

[20] Piezo Kinetics Corporation, (814) 355-1593, personal communications.

[21] J.H. Robinson, Acoustics Division, NASA Langley Research Center, personal communications.

[22] J.H. Robinson, S.A. Rizzi, S.A. Clevenson and E.F. Daniels, "Large Deflection Random Response of Flat and Blade Stiffened Carbon Panels," Proceedings of the $33^{\text {rd }}$ AIAA/ASME/ASCE/AHS/ASC Structures, Structural Dynamics and Materials Conference, Dallas, TX, 1992.

[23] R.C. Smith, "A Galerkin Method for Linear PDE Systems in Circular Geometries with Structural Acoustic Applications," SIAM Journal on Scientific Computing, to appear. 